\title{
Milk And Resistance Exercise: What Are The Real Factors That Can Limit The Intake?
}

\author{
Humberto Nicastro ${ }^{1 *}$, Gustavo Barquilha ${ }^{1}$, Carlos Tomaiolo ${ }^{1}$, Natalia Marques ${ }^{2}$, Euclésio Bragança ${ }^{1}$ \\ ${ }^{1}$ IntegralMedica Suplementos Nutricionais - Av. Cidade Jardim, 400, Jardim Europa PO Box 01454-000 - São Paulo, SP - Brazil. \\ ${ }^{2}$ Universidade Federal do Estado de São Paulo - São Paulo, SP, Brazil
}

\begin{abstract}
Received: March 19, 2016; Accepted: April 10, 2016; Published: May 13, 2016
*Corresponding author: Humberto Nicastro Integral Medical Suplementos Nutricionais - Av. Cidade Jardim, 400, Jardim Europa, PO Box 01454-000 - São Paulo, SP - Brazil. Tel/Fax.: +55 11 3254-0444; E-mail: nicastroh@yahoo.com.br
\end{abstract}

\begin{abstract}
Sports nutrition is a dynamic area that generates new concepts and data every day. The reason for this is the potential that nutrients have to improve performance through energy provision, recovery, attenuation of muscle damage and pain, prevent the occurrence of injuries, etc. All of these aspects are related to skeletal muscle metabolism. Some foods can provide specific nutrients in the correct form and amount in order to contribute to muscle metabolism requirements. In this concept, milk represents an interesting food that has demonstrated positive and significant results in muscle mass and strength when associated with resistance exercise in humans. In contrast, there are some discussions about milk intake that go against the well-documented effects relatedto sports science. The arguments used in such discussions mainly include: digestibility, intolerance, allergenic potential, and aspects related to its composition. Thus, can milk be considered an ergogenic food or a risk in improvements of skeletal muscle metabolism?
\end{abstract}

Keywords: Whey; Casein; Protein synthesis; Exercise; Strength; Hypertrophy

\section{Introduction}

Nutritional supplements have been emerged as potential ergogenic aids that can improve exercise performance. Among the exercise-induced physiological adaptations, skeletal muscle hypertrophy and strength, delayed onset muscle soreness and pain, muscle damage, tolerance to fatigue,and acidosis (commonly expressed as time-to-exhaustion and decrease in time-trial tests) are the main responses that can be improved through the intake of some nutritional supplements. Therefore, it is plausible to assume that some compounds are able to improve resistance exercise-induced muscle adaptations, while others are directed to intermittent and/or aerobic exercises. Although there are significant differences among the exercise variables according to the type of the exercise, several studies and meta-analyses have suggested that the main route by which the majority of nutritional compounds act in order to promote ergogenic effects are related to exercise volume or to post-translational muscle signaling [1-3].
Regarding resistance exercise, muscle mass accretion and strength are the main physiological adaptations observed in response to a training program. Skeletal muscle hypertrophy can be defined as the accumulation of contractile proteins that results in the increase of sarcomeres size, while strength is defined as the capacity of the muscle to produce force [4]. These adaptations are coordinated by several exercise variables that include: volume (number of exercise, sets, and repetitions), intensity, rest intervals, and frequency. Among these, it has been accepted that the most responsive variable depending on the nutritional stimuli appears to be the exercise volume. Thus, it is possible to assume that some nutrients are able to increase resistance exercise volume (repetitions) that, chronically, can induce an increase in muscle mass and strength. Basically, these are compounds that have phosphates in their structure to provide energy or can delay exercise-induced muscle acidosis, such as creatine and betaalanine, respectively [2, 5].

However, Essential Amino Acids (EAA) are potential ergogenic supplements that can improve muscle hypertrophy and strength and do not present phosphates in their structure neither can attenuate muscle acidosis. The increase in EAA availability has been described to optimize skeletal muscle post-translational modifications induced by resistance exercise, i.e. phosphorylation through a calcium-dependent process, which can result in the increase of the efficacy and efficiency of ribosomal translation of contractile proteins (actin, myosin, etc.) [6]. Therefore, EAA intake stimulates resistance exercise-induced muscle protein synthesis through translation initiation of the messenger RNA (mRNA) that can result in the accumulation of contractile proteins and, in long term, it is expected that muscle strength can also be increased in response to muscle hypertrophy. The most known and studied supplement able to provide EAA (with the adequate amount of leucine, which will be discussed later) is the protein obtained from milk fraction called whey protein $[7,8]$.

Recently, some research groups have demonstrated that some foods can also induce a similar response of whey protein supplementation on resistance exercise-induced muscle 
adaptations. Milk presents an interesting composition regarding EAA and has emerged as a potential ergogenic food able to increase muscle mass and strength in humans. Some authors have classified milk as a "sports drink" because of its ergogenic potential in humans [9]. However, some studies discuss the possible adverse effects of milk intake in humans because of its allergenic potential, low capacity to digest protein subfractions, and intolerance presented by some individuals. Thus, the aim of the present narrative review is to discuss the physiological implications of milk intake in humans in terms of adverse effects such as digestibility, allergy, intolerance and the resistance exercise-related benefits. The origin of both of positive and negative evidences as well as the experimental design of the studies will be discussed in order to provide to the reader information to be used in clinical practice and to exercise the critical sense about the evidences.

\section{Human's digestibility to milk proteins fractions}

Cow`s milk is basically composed of water, lipids, carbohydrates, proteins, vitamins and minerals, such calcium and phosphorus. Although there are many process that can improve the quality of milk composition - i.e., addition of vitamins A and D -, it is supported by numerous studies that cow's milk composition may differ according to many factorsincluding genetic, stage of lactation, ruminal fermentation, udder infections, production and processing practices, seasonal and regional differences and mainly, regimen feed [10-14].The regimen feed exerts substantial implications on milk composition;n, especially regarding to the content of fatty acids - cow which were fed predominantly with fodder has distinct fatty acid profile than milk from cows which were fed with seeds and grains $[15,16]$.

The lipid content of cow's milk is made up of over 400 distinct species of fatty acids, being considered the most complex fat present in the human diet. Source of saturated fatty acids, the milk fat also contains smaller amounts of butyric acid, palmitoleic acid, conjugated linoleic acid, and phytanic acid. Although they constitute only a small percentage of fatty acids present in fat milk, it is suggested that these may still be biologically relevant[14] The main carbohydrate found in cow's milk is lactose which represents about $5 \%$ of milk total components. Regarding protein content, milk is composed of two major types of proteins with different amino acid profiles: casein (protein phosphate divided into four major classes - alpha s1, alpha s2, beta and kappa) and soluble proteins (also known as whey, represented by betalactoglobulin, alpha-globulin, serum albumin, immunoglobulins, lactoferrin and other minor fractions with high concentrations of sulfur amino acids) [17]. Some factors are associated with variations in the amount of proteins in cow's milk - as cows became older, the proportion of casein decreases [18].

The protein digestion is a complex process in which occurs exchange of proteins, peptides, amino acids, urea, and ammonia from the intestinal lumen to systemic sites [19, 20]. After this process, the hydrolysis of these proteins through the action of enzymes such as trypsin, chymotrypsin, pepsin, thermolysin, pancreatin, carboxypeptidases and endopeptidases occurs, releasing oligopeptides, dipeptides, tripeptides and free amino acids for intestinal absorption [19, 21]. Milk proteins present 95$96 \%$ of digestibility and $74 \%$ of protein postprandial utilization on average [22, 23].

To the best of our knowledge, the most well controlled studied evaluating this question was conducted by Boutrou et al. [19]. In a very elegant study, the authors determined the kinetics formation of peptides in the jejunum after intake of two protein sources. Volunteers were equipped with a double-lumen nasogastric tube to the proximal jejunum and ingested $30 \mathrm{~g}$ of ${ }^{15} \mathrm{~N}$-labeled casein or whey protein. Beta-casein was the main peptides precursor founded. The authors observed a total of 356 and 146 peptides in the proximal jejunum after casein and whey intake, respectively. However, these data represent only the partial result of protein digestion and cannot be assumed as indigestibility of milk proteins. It is important to consider that these peptides can suffer hydrolysis by aminopeptidases present in the enterocytes posteriorly. Furthermore, the authors conclude that it must be investigated if these peptides have or not biological activity.

Although some professionals argue that humans cannot digest some fraction of milk proteins, it is important to emphasize that there are no well controlled clinical studies confirming such information in humans. Studies that demonstrate the deleterious effects of milk intake in the gastrointestinal tract come from experimental models (mainly rodents). However, it is important to emphasize that rodents is a specie that did not evolve to milk intake and do not follow the same dietary and environmental pattern than humans. Therefore, it not possible to extrapolate experimental evidences to the clinical practice. We can assume other factor like milk composition and lifestyle (discussed later) can be considered crucial when relating milk with health side effects.However, the data presented by Boutrou et al. [19] should be considered as the first evidence that human digestibility of milk fractions it is an important point that should be addressed in future studies.

Since it is not the focus of the present review to discuss the lack of evidences in humans, we will consider if milk intake promote or not ergogenic effects in humans when submitted to resistance exercise. Several reports have described that the limiting factor to protein intake-induced muscle hypertrophy and strength is the aminoacidemia pattern, especially leucine threshold (leucinemia).Thus, despite of digestibility, the major concern of milk intake to optimize resistance exercise-induced muscle adaptations is the possibility achieve the ideal pattern of aminoacidemia (and leucinemia).

\section{Intolerance and allergenic potential: clinical evidence}

Although cow's milk allergy and cow's milk intolerance are two different terms, they are often used interchangeably, resulting in confusion both in clinical practice and in research reports [24]. So to address this issue, we define them. Cow's milk allergy is an immunologically mediated reaction to cow's milk proteins that may involve the gastrointestinal tract, skin, respiratory tract, or multiple systems, i.e, systemic anaphylaxis. It present 
heterogeneity and is closely related to the immunological and clinical phenotype [25]. Its prevalence in the general population is probably 1 to $3 \%$, being highest in infants and lowest in adults. Even though it can cause severe morbidity and even fatality [24].

The immune response arising from the intake of milk is directly related to the types of protein. For over two decades, there are publications with milk allergy, most showing effects in children during the first two years. Host demonstrated in a cohort of 1749 newborns that $2.2 \%$ developed allergy/intolerance to cow's milk, and all of which had ingested cow's milk formula $(40-830 \mathrm{~mL})$ neonatally while $12 \%$ without supplements of cow's milk formula did not develop the same symptoms. The main conclusion is early neonatal cow's milk protein intake is probably a condition of sensitization and development of later adverse reactions, both intolerance as allergy [26].

Consumption of cow's milk and immune responses has also been described. Mostly, cow's milk allergy is caused by IgEmediated (type I) reactions, but evidence for type III (immune complex) reactions and type IV (cell mediated reactions) have been demonstrated and reviewed by Host [27, 28]. More recently, it was demonstrated in cow's milk-sensitive esophagitis that there is dense infiltrate of eosinophils and increased $\mathrm{T}$ cell activation with upregulation of the chemokine eotaxin. In cow's milk-sensitive enteropathy, there is T cell activationwhich often results as a sequel of gastroenteritis.There is evidence of reduced Th1 response in these children, even those who were breastfed but who were not sensitized by the components of breast milk in response to food intake of the mother. This is maybe related to IgA deficiency and low levels of cytokine transforming growth factor beta [29]. Despite the lack of studies in adults with this approach, it is possible to speculate a potential correlation between these responsestriggered in childhood that may be responsible for the symptoms in adults. However, such information must be studied and confirmed. The prognosis of cow's milk allergy is good with a remission rate about $45-50 \%$ at one year, $60-75 \%$ at two years, and $85-90 \%$ at three years. Associated adverse reactions to other foods especially egg, soy, peanut and citrus represents about $41-54 \%$, and allergy against inhalants is about $50-80 \%$ before puberty [27].

Considering the previous information described, the characterization of allergy to cow's milk as prevention and treatment have been defined and is supported by the American Academy of Pediatrics' and the European Society of Pediatric Gastroenterology, Hepatology and Nutrition recommendations [30]. But the process of re-introduction of allergenic proteins in adults remains a topic of debate[25].

On the other hand, cow's milk intolerance should refer to nonimmunologic reactions, such as disorders of digestion, absorption, or metabolism of some components [24]. Food intolerance, may be defined as a reproducible adverse reaction to the ingestion of a food or to any of its components, i.e. proteins, carbohydrates,fats, and additives, and includes toxicand metabolic reactions [29]. The most common cause when considering the consumption of cow's milk is lactase enzyme deficiency, which is mostly acquired during late childhood or adulthood [24]. It appears to be more prevalent in dark-skinned populations and lowest in northern Europeans. For example, lactose tolerance is exceptionally widespread in Northern European countries such as Sweden and Finland, with tolerance levels of $74 \%$ and $82 \%$, respectively. In a survey of a population-representative sample of 2251 Canadians aged $\geq 19$ years conducted by Barr [31], 16\% self-reported lactose intolerance. This was more common in women and in nonwhitesand less common in those $>50$ years of age. This latter finding is surprising, since normally the organic aging process generates a decrease in enzyme production.

Unlike cow's milk allergy, most research of intolerance to cow's milk is related to specific diseases and disorders in the gastrointestinal tract. For example, irritable bowel syndrome patients with diarrhea often report milk intolerance, most of all because lactose intolerance. Gas production and visceral hypersensitivity both contribute to digestive symptoms, especially bloating and borborygmi, in irritable bowel syndrome patients after lactose ingestion [32]. But in this case, the symptoms are not limited to the gastrointestinal tract. Patients can be characterized by anxiety, mucosal immune activation and increased visceral sensitivity after lactose ingestion [33].

Although double-blind studies show that lactose-intolerant individuals can consume moderate quantities of milk products without perceptible symptoms, many who perceive that are lactose intolerant should limit or avoid milk products, which can potentially compromise calcium and vitamin D intakes [31]. Therefore, the nutritional control should always be prioritized, regardless of the presence of allergy or intolerance, respecting the individuality and polymorphisms of patients.

\section{Milk and sports performance}

\section{Acute effects on resistance exercise}

Although there are few acute evidences evaluating the effects of milk intake associated with resistance exercise, the available studies were performed in a well-controlled design with gold standard methodologies, which implies in strong reliability of the data. It is important to emphasize that acute data does not necessarily reflects a chronic result because of the limitations imposed by the experimental design (for example, exercise in the post-absorptive state), but suggests potential long-term implications in terms of effectiveness and safety.

In a very elegant study, Wilkinson et al. [34] examined the effects of consuming an isonitrogenous, isoenergetic, and macronutrient-matched soy or milk beverages on skeletal muscle protein balance after resistance exercise in healthy young men in a randomized and single-blinded fashion. In the morning after an overnight fast, subjects consumed a standard beverage and performed 4 sets of 10 repetitions of a standardized unilateral leg workout (leg press, hamstring curl, and knee extension) at $80 \%$ of 1 Repetition Maximum (1RM) and then ingested a 500-ml drink contained either nonfat milk or soy. The authors measured skeletal muscle protein synthesis through the infusion of the radioisotope $\mathrm{L}-\left[1-{ }^{13} \mathrm{C}\right]$ leucine before and after 180 minutes of the 
exercise protocol. Both interventions resulted in a positive net protein balance. However, milk intake promoted greater protein balance after exercise $(p<0.05)$. Similarly, skeletal muscle protein synthesis rate was also significantly increased after milk intake when compared to the soy consumption $\left(0.10 \pm 0.01 \% \cdot \mathrm{h}^{-1}\right.$ vs. $\left.0.10 \pm 0.07 \% \cdot h^{-1}\right)$. Therefore, milk intake promotes significantly increase in muscle mass accretion after resistance exercise which suggests that, chronically, skeletal muscle hypertrophy and increase of strength could be expected.

Recently, Cockburn et al. [35] evaluated the effects of a carbohydrate-protein milk supplement on exercise-induced muscle damage. In a randomized design, healthy male subjects who competed in semiprofessional soccer were submitted to a bout of 6 sets of 10 repetitions of unilateral eccentric-concentric knee flexions at $1.05 \mathrm{rad}_{\mathrm{s}} \mathrm{s}^{-1}$ and consumed $500 \mathrm{ml}$ of semiskimmed milk or water. Although no differences were observed in blood markers (creatine kinase and myoglobin; $p>0.05$ ) and in delayed onset muscle soreness during 72 hours of the intervention $(p>0.05)$, milk intake demonstrated a possible benefit for limiting increases in 10-m sprint time 48 hours after the exercise protocol. Furthermore, a likely benefit of attenuating increases in 15-m sprint time was observed 48 and 72 hours and for attenuation of increases in agility time 72 hours after the intervention.

It should be emphasize that in both studies the authors did not describe any exclusion criteria adopted for the volunteers, which implies a probability of milk-intolerant and -allergic subjects were included in the study. Additionally, no exclusions of subjects were reported because of adverse effects. Such data corroborate the recent perspective of Melnik et al. [36] that the metabolic effects of milk protein intake (including the adverse effects) have to be regarded in the context of the individual's pre-existing metabolic (obesity, insulin resistance) and exercise status (sedentary life style).

\section{Chronic effects on resistance exercise}

Since acute studies provide potential results of milk intake regarding resistance exercise-induced muscle adaptations, chronic studies are the second the step needed to confirm such information with greater external validity. The studies described below are considered well controlled in terms of methodological tools and are conducted in order to extrapolate the data to the real world. This is quite important when we consider milk intake because there are several arguments that are discussed against its benefits but that were not well studied or evaluated. It is important to emphasize that in these studies described the subjects were not submitted to dietary control, i.e. maintained their habitual food intake. Thus, the absence of adverse effects and ergogenic results observed can just be attributed to the exercise program associated with milk intake.

The same study of Wilkinson et al. [34] was posteriorly conducted in a randomized chronic design by the same group [37]. The subjects performed 12 weeks of rotating split-body resistance exercise 5 days/week and consumed 1 hour after each exercise bout fat-free milk, fat-free soy protein or maltodextrin.
Both protein interventions were isoenergetic, isonitrogenous, and macronutrient ratio matched. In this chronic study, subjects who reported being lactose intolerant or having any history of milk protein allergy were not allowed to participate. Fat-free milk intake resulted in greater increase in type 2 muscle fiber when compared to other groups $(p<0.05)$ and in type 1 muscle fibers when compared to the maltodextrin group ( $<0.05)$. Fat- and bone-free mass also showed greater increase in milk group when compared to the others $(\mathrm{p}<0.05)$. No significant differences were observed in strength measure among groups ( $p>0.05$ ). These results confirm the acute data presented by Wilkinson et al. [34] demonstrating that, in this case, skeletal muscle protein synthesis is a strong predictor of muscle hypertrophy and that fat-free milk intake represent a potential nutritional strategy to improve resistance-exercise induced muscle adaptations for adult male individuals that do not present restrictions to milk intake.

Josse et al. [38] randomized young women to drink either fat-free milk or isoenergetic carbohydrate immediately after and 1 hour after exercise $(500 \mathrm{ml} /$ dose $)$. The subjects followed the same exercise regimen proposed by Hartman et al. [37]. Those diagnosed with lactose intolerance or had any history of a milk protein allergy were excluded from participating in the study. The results pattern observed were quite similar to that observed by Hartman et al. [37]. Lean body mass increased more significantly in the milk group and compared to the placebo $(\mathrm{p}<0.01)$ and fat mass decreased only in the milk group ( $<<0.01)$. However, contrary to the findings of Hartman et al. [37], isotonic strength increased more in the milk group when compared to the placebo $(p<0.05)$. Again, these data clearly demonstrate that milk has a strong ergogenic potential to be considered during resistance exercise training programs for men and women that do not present restriction to milk intake.

In a randomized design, Josse et al. [39] also evaluated the effects of resistance and/or aerobic exercise associated with hypoenergetic diet varying in protein and calcium content from dairy foods on weight and fat mass loss and lean mass gain in overweight and obese premenopausal women. Subjects were randomized in 3 groups which received high protein and high dairy (30 and 15\% of energy), adequate protein and moderate dairy ( 15 and $7.5 \%$ of energy), and adequate protein and low dairy (15 and $<2 \%$ of energy) during 16 weeks. The dairy products used to control dairy and calcium intake were: chocolate milk, source yogurt, white milk, and cheddar cheese. Interestingly, this study described the inclusion and exclusion criteria. Low dairy products intake, sedentary life style and regular menstrual cycle were considered inclusion criteria, while metabolic risk factors (cholesterol, insulin, and glucose concentrations and blood pressure), clinically diagnosed dairy protein allergy and lactose intolerance were adopted as exclusion criteria. The results demonstrated that all the groups lost body weight and fat, but high protein and high dairy intake group presented a greater weight, visceral adipose tissue, and trunk fat loss when compared to the others during 8-16 weeks $(\mathrm{p}<0.05)$. Furthermore, high protein and high dairy intake group gained significantly more lean body 
mass when compared to the others ( $\mathrm{p}<0.05)$. Therefore, it is possible to conclude based on such well-controlled data that the chronic dairy intake associated with exercise promotes significant reduction in body weight and adipose tissue and increase in lean body mass in female premenopausal subjects that are not allergic to dairy protein and lactose intolerant.

\section{Conclusion}

Although evidence shows that milk consumption has beneficial effects on physical exercise, some parameters must be considered. First of all, biochemical individuality must be evaluated when prescribing any food or supplement Professionals should follow the nutritional conduct and guide the best form of consumption according to well-controlled clinical trials. Thus, food intolerance or allergies should be evaluated previously to the recommendation.

It is also important to evaluate the gastrointestinal function in order to minimize some disturbances. In some comes, milk or dairy products intake may be reduced or disallowed. Furthermore, the professional must be aware of other conditions that may interfere with intestinal metabolism, such as lifestyle in which the individual is exposed (stress, inadequate nutrition, excessive physical activity, sedentarism, insulin resistance, among others).

\section{References}

1. Cermak NM, Res PT, de Groot LC, Saris WH, and van Loon LJ. Protein supplementation augments the adaptive response of skeletal muscle to resistance-type exercise training: a meta-analysis. Am J Clin Nutr. 2012;96(6):1454-1464. doi: 10.3945/ajcn.112.037556

2. Hobson RM, Saunders B, Ball G, Harris RC, and Sale C. Effects of betaalanine supplementation on exercise performance: a meta-analysis. Amino Acids. 2012;43(1):25-37. doi:10.1007/s00726-011-1200-z

3. Hoon MW, Johnson NA, Chapman PG, and Burke LM. The effect of nitrate supplementation on exercise performance in healthy individuals: a systematic review and meta-analysis. Int J Sport Nutr Exerc Metab. 2013;23(5):522-532

4. Zanchi NE and Lancha AH Jr. Mechanical stimuli of skeletal muscle: implications on mTOR/p70s6k and protein synthesis. Eur J Appl Physiol. 2008;102(3):253-263. doi:10.1007/s00421-007-0588-3

5. Harris RC, Soderlund K, and Hultman E. Elevation of creatine in resting and exercised muscle of normal subjects by creatine supplementation. Clin Sci (Lond). 1992;83(3):367-374

6. Gulati P, Gaspers LD, Dann SG, Joaquin M, Nobukuni T, Natt F, et al. Amino acids activate mTOR complex 1 via $\mathrm{Ca} 2+/ \mathrm{CaM}$ signaling to hVps34. Cell Metab. 2008;7(5):456-465. doi:10.1016/j. cmet.2008.03.002

7. Nicastro H, Artioli GG, Costa Ados S, Solis MY, da Luz CR, Blachier F, et al. An overview of the therapeutic effects of leucine supplementation on skeletal muscle under atrophic conditions. Amino Acids. 2011;40(2):287-300. doi: 10.1007/s00726-010-0636-x

8. Nicastro H, Zanchi NE, da Luz CR, de Moraes WM, Ramona P, de Siqueira Filho, et al. Effects of leucine supplementation and resistance exercise on dexamethasone-induced muscle atrophy and insulin resistance in rats. Nutrition. 2012;28(4):465-471. doi:10.1016/j. nut.2011.08.008
9. Roy BD. Milk: the new sports drink? A Review. J Int Soc Sports Nutr. 2008;5-15. doi:15. 10.1186/1550-2783-5-15

10.Sutton JD, Altering Milk Composition by Feeding. Journal of Dairy Science. 1989;72(10):2801-2814

11. Jami E, White BA, and Mizrahi I. Potential role of the bovine rumen microbiome in modulating milk composition and feed efficiency. PLoSOne. 2014;9(1):e85423. doi:10.1371/journal.pone.0085423

12. Palmquist DL, Beaulieu AD, and Barbano DM. Feed and animal factors influencing milk fat composition. J Dairy Sci. 1993;76(6):1753-1771

13. Dhiman TR, Nam SH, and Ure AL. Factors affecting conjugated linoleic acid content in milk and meat. Crit Rev Food Sci Nutr. 2005;45(6):463482.doi:10.1080/10408390591034463

14. Kratz M, Baars T, and Guyenet S. The relationship between highfat dairy consumption and obesity, cardiovascular, and metabolic disease. EurJNutr. 2013;52(1):1-24. doi:10.1007/s00394-012-0418-1

15. Leiber F, Kreuzer M, Nigg D, Wettstein HR, and Scheeder MR. A study on the causes for the elevated n-3 fatty acids in cows' milk of alpine origin. Lipids. 2005;40(2):191-202

16. Couvreur S, Hurtaud C, Marnet PG, Faverdin P, and Peyraud JL. Composition of milk fat from cows selected for milk fat globule size and offered either fresh pasture or a corn silage-based diet. J Dairy Sci. 2007;90(1):392-403. doi:10.3168/jds.S0022-0302(07)72640-1

17. Lacroix M, Bos C, Leonil J, Airinei G, Luengo C, Dare S, et al. Compared with casein or total milk protein, digestion of milk soluble proteins is too rapid to sustain the anabolic postprandial amino acid requirement. Am J Clin Nutr. 2006;84(5):1070-1079

18. Ng-Kwai-Hang KF, Hayes JF, Moxley JE, and Monardes HG. Environmental influences on protein content and composition of bovine Milk. J Dairy Sci. 1982;65(10):1993-1998. doi:10.3168/jds. S0022-0302(82)82449-1

19. Boutrou R, Gaudichon C, Dupont D, Jardin J, Airinei G, Marsset-Baglieri A, et al. Sequential release of milk protein-derived bioactive peptides in the jejunum in healthy humans. Am J Clin Nutr. 2013;97(6):13141323. doi:10.3945/ajcn.112.055202

20. Nasset ESJ, J.S.. Mixture of endogenous and exogenous protein in the alimentary tract. J Nutr. 1961;74(4):461-465.

21. Choi JS, L., Hassan, A.,and Anand, S..Bioactive peptides in dairy productsBioactive peptides in dairy products. Int J Dairy Technol. 2012;65(1):1-12 doi: 10.1111/j.1471-0307.2011.00725.x

22. Bos C, Mahe S, Gaudichon C, Benamouzig R, Gausseres N, Luengo C,et al. Assessment of net postprandial protein utilization of $15 \mathrm{~N}$-labelled milk nitrogen in human subjects. Br J Nutr. 1999;81(3):221-226

23. Morens C, Bos C, Pueyo ME, Benamouzig R, Gausseres N, Luengo C, et al. Increasing habitual protein intake accentuates differences in postprandial dietary nitrogen utilization between protein sources in humans. J Nutr. 2003;133(9):2733-2740

24. Bahna SL. Cow's milk allergy versus cow milk intolerance. Ann Allergy Asthma Immunol. 2002;89(6 Suppl 1):56-60

25. Nicolaou N, Tsabouri S, and Priftis KN. Reintroduction of Cow's Milk in Milk-Allergic Children. Endocr Metab Immune Disord Drug Targets. 2014;14(1):54-62.

26. Host A. Importance of the first meal on the development of cow's milk allergy and intolerance. Allergy Proc. 1991;12(4):227-232

27. Host A. Cow's milk protein allergy and intolerance in infancy. Some clinical, epidemiological and immunological aspects. Pediatr Allergy 


\section{Immunol. 1994;5(5 Suppl):1-36}

28. Host A, Jacobsen HP, Halken S, and Holmenlund D. The natural history of cow's milk protein allergy/intolerance. Eur J Clin Nutr. 1995;49(Suppl 1):S13-18

29. Walker-Smith J. Cow's milk allergy: a new understanding from immunology. Ann Allergy Asthma Immunol. 2003;90(6 Suppl 3):8183

30. Grimshaw KE, Maskell J, Oliver EM, Morris RC, Foote KD, Mills EN,et al. Introduction of complementary foods and the relationship to food allergy. Pediatrics. 2013;132(6):e1529-1538. doi:10.1542/ peds.2012-3692

31. Barr SI. Perceived lactose intolerance in adult Canadians: a national survey. Appl Physiol Nutr Metab. 2013;38(8):830-835. doi:10.1139/ apnm-2012-0368

32. Zhu Y, Zheng X, Cong Y, Chu H, Fried M, Dai N, et al. Bloating and distention in i rritable bowel syndrome: the role of gas production and visceral sensation after lactose ingestion in a population with lactase deficiency. Am J Gastroenterol. 2013;108(9):1516-1525. doi:10.1038/ajg.2013.198

33. Yang J, Deng Y, Chu H, Cong Y, Zhao J, Pohl D, et al. Prevalence and presentation of lactose intolerance and effects on dairy product intake in healthy subjects and patients with irritable bowel syndrome. Clin Gastroenterol Hepatol. 2013;11(3):262-268e1. doi:10.1016/j. cgh.2012.11.034

34. Wilkinson SB, Tarnopolsky MA, Macdonald MJ, Macdonald JR
Armstrong D, and Phillips SM. Consumption of fluid skim milk promotes greater muscle protein accretion after resistance exercise than does consumption of an isonitrogenous and isoenergetic soyprotein beverage. Am J Clin Nutr. 2007;85(4):1031-1040

35. Cockburn E, Bell PG, and Stevenson E. Effect of milk on team sport performance after exercise-induced muscle damage. Med Sci Sports Exerc. 2013;45(8):1585-1592. doi:10.1249/MSS.0b013e31828b7dd0

36. Melnik BC, Schmitz G, John S, Carrera-Bastos P, Lindeberg S, and Cordain L. Metabolic effects of milk protein intake strongly depend on pre-existing metabolic and exercise status. Nutr Metab (Lond). 2013;10(1):60.doi:10.1186/1743-7075-10-60

37. Hartman JW, Tang JE, Wilkinson SB, Tarnopolsky MA, Lawrence RL, Fullerton AV, et al. Consumption of fat-free fluid milk after resistance exercise promotes greater lean mass accretion than does consumption of soy or carbohydrate in young, novice, male weightlifters. Am J Clin Nutr. 2007;86(2):373-381

38. Josse AR, Tang JE, Tarnopolsky MA, and Phillips SM. Body composition and strength changes in women with milk and resistance exercise. Med Sci Sports Exerc. 2010;42(6):1122-1130. doi:10.1249/ MSS.0b013e3181c854f6

39. Josse AR, Atkinson SA, Tarnopolsky MA, and Phillips SM. Increased consumption of dairy foods and protein during diet- and exerciseinduced weight loss promotes fat mass loss and lean mass gain in overweight and obese premenopausal women. J Nutr. 2011;141(9):1626-1634. doi:10.3945/jn.111.141028 